\title{
Du nouveau dans la maladie d'Alzheimer
}

Les mécanismes pathogéniques de la maladie d'Alzheimer s'éclairent de toutes parts. Dans les formes familiales de cette démence dont la prévalence dans la population âgée est si élevée qu'elle pose un grave problème de santé publique [1], on savait que les mutations dans le gène de la préséniline 1 (PS1) favorisaient l'accumulation du peptide $\beta$ amyloïde mais on n'avait pas la preuve qu'elles intervenaient dans le routage de la protéine APP $\left(\mathrm{m} / \mathrm{s} n^{\circ} 1\right.$, vol. 13 , p. 106). Sur des cultures de neurones d'embryons de souris déficientes en $P S 1$, il vient d'être démontré que l'absence de PS1 empêche le clivage $\mathrm{du}$ domaine transmembranaire de l'APP par la $\gamma$ sécrétase, avec accumulation de fragments carboxy-terminaux d'APP et, en même temps, provoque une chute spectaculaire du peptide $\beta$ amyloïde (cinq fois moins) [2]. Ainsi, les mutations de PS1 doivent correspondre à un gain de fonction et le blocage de l'activité PS1 pourrait être utilisé dans la maladie d'Alzheimer en thérapeutique pour diminuer l'accumulation de la substance amyloïde. Cette observation est corroborée par l'étude de souris porteuses de deux transgènes (un mutant $A P P$ et un mutant PS1) chez lesquelles des dépôts de substance amyloïde s'accumulent rapidement dans les régions cérébrales électivement atteintes dans la maladie d'Alzheimer [3]. La compréhension des formes sporadiques vient, elle aussi, de progresser dans deux directions. Une équipe hollandaise vient de découvrir deux protéines anormales chez des malades atteints de maladie d'Alzheimer et chez des trisomiques 21 âgés [4]. Elles sont la conséquence d'un mécanisme mutationnel original que cette même équipe avait observé antérieurement dans le gène de la vasopressine chez les rats Brattleboro [5]: dans certains ARNm, il existe des suites de motifs GAGAG dont deux nucléotides GA tendent à se perdre, particulièrement dans les cellules vieillissantes, ce qui modifie le cadre de lecture. Or, ces motifs sont présents dans les ARNm des gènes codant pour l'APP et pour l'ubiquitine $\mathrm{B}$, toutes deux impliquées dans la maladie d'Alzheimer. Des protéines mutantes dans leur région carboxy-terminale, conséquences de délétions GA, furent retrouvées dans les plaques séniles de cerveaux de malades, de trisomiques et de sujets âgés, alors qu'elles ne furent jamais observées dans des cerveaux de sujets jeunes décédés de causes diverses. Enfin, dans deux groupes de population, l'une hispanique et l'autre d'Amérique du Nord, la prédisposition à présenter une maladie d'Alzheimer dépendrait d'un polymorphisme $\mathrm{A} / \mathrm{T}$ dans la région régulatrice du gène $A P O E$ en position -491 [6]. Les sujets homozygotes AA auraient un risque significativement augmenté de développer la maladie (multiplié par 4), et ce, indé- pendamment de l'allèle APOE4 qui était mis en avant jusqu'à présent. Spécifiquement, l'allèle A-491 serait associé à une augmentation de l'activité constitutive du promoteur APOE, se traduisant par une augmentation de la concentration d'APOE dans le cerveau. Les groupes de recherche sur cette maladie si préoccupante, on le voit, ne chôment pas, et les lecteurs de médecine/sciences seront scrupuleusement tenus au courant des développements ultérieurs de ces résultats préliminaires.

S.G.

\footnotetext{
1. Ritchie K, Robine JM. La démence sénile en France: une estimation de la charge actuelle et projection des tendances futures. Med Sci 1994; $10: 680-6$.

2. de Strooper B, Saftig P, Craessaerts K, Vanderstichele H, Guhde G, Annaert W, Von Figura K, Van Leuven F. Deficiency of presenilin-1 inhibits the normal cleavage of amyloid precursor protein. Nature $1998 ; 391$ : 387.

3. Holcomb L, Gordon MN, McGowan E, Yu X, Benkovic S, et al. Accelerated Alzheimer-type phenotype in transgenic mice carrying both mutant amyloid precursor protein and presenilin-1 transgenes. Nat Med 1998; 4: 97-100.

4. van Leeuwen F, de Kleijn DPV, van den Hurk H, Neubauer A, Sonnemans MAF, et al. Frameshift mutants of $\beta$ amyloid precursor protein and ubiquitin-B in Alzheimer's and Down patients. Science 1998 ; 279 : 242-7.

5. Evans DAP, van der Kleij AA, Sonnemans MA, Burbach JP, van Leeuwen FW. Frameshift mutations at two hotspots in vasopressin transcripts in post-mitotic neurons. Proc Natl Acad Sci USA 1994; 91: 6059-63.

6. Bullido MJ, Artiga MJ, Recuero M, Sastre I, Garcia MA, et al. A polymorphism in the regulatory region of $A P O E$ associated with risk fir Alzheimer's dementia. Nat Genet 1998; 18: 69-71.
}

\section{CONFÉRENCES JACQUES MONOD 1998 PLASTICITÉ ET ADAPTATION DE LA MOTRICITÉ : Ontogenèse, apprentissage moteur et restauration fonctionnelle AUSSOIS (France) - 21-25 septembre 1998 \\ Président: $\quad$ MASSION Jean. C.N.R.S., Laboratoire de Neurobiologie et Mouvements, 31, chemin Joseph-Aiguier, F-13402 Marseille Cedex 20, France. Phone - Téléphone : + 33491 16 4150 - Fax- Télécopie : + 334917750 84. E-mail - Courrier électronique : massion@lnf.cnis-mrs.fr Armand J., Assaiante C., Berthoz A., Champagnat.., Clarac F., Dietz V., Forssberg H., Freund H.J., Gauhier G. Gauthier P, Glover J., Gramsberger A., Graybiel A., Hallett M., HuthbornH. loffe M. Jeannerod M. Kawato M., Latash M., Massion J., Nieoullon A., Nudo R., Pettersson L.G., Rispal-Padel L., Rossignol S., Sillar K., Simmers J., Thelen E., Wiesendanger M. DATE LIMITE D'INSCRIPTION : 30 AVRIL 1998}

